\title{
GCU
}

Glasgow Caledonian

University

University for the Common Good

\section{Assessing and augmenting SCADA cyber security: a survey of techniques}

Nazir, Sajid; Patel, Shushma; Patel, Dilip

Published in:

Computers \& Security

DOI:

10.1016/j.cose.2017.06.010

Publication date:

2017

Document Version

Author accepted manuscript

Link to publication in ResearchOnline

Citation for published version (Harvard):

Nazir, S, Patel, S \& Patel, D 2017, 'Assessing and augmenting SCADA cyber security: a survey of techniques', Computers \& Security, vol. 70, pp. 436-454. https://doi.org/10.1016/j.cose.2017.06.010

\section{General rights}

Copyright and moral rights for the publications made accessible in the public portal are retained by the authors and/or other copyright owners and it is a condition of accessing publications that users recognise and abide by the legal requirements associated with these rights.

Take down policy

If you believe that this document breaches copyright please view our takedown policy at https://edshare.gcu.ac.uk/id/eprint/5179 for details of how to contact us. 


\title{
Assessing and Augmenting SCADA Cyber Security-A Survey of Techniques
}

\author{
S. Nazir, S. Patel, D. Patel
}

\begin{abstract}
SCADA systems monitor and control critical infrastructures of national importance such as power generation and distribution, water supply, transportation networks, and manufacturing facilities. The pervasiveness, miniaturisations and declining costs of internet connectivity have transformed these systems from strictly isolated to highly interconnected networks. The connectivity provides immense benefits such as reliability, scalability and remote connectivity, but at the same time exposes an otherwise isolated and secure system, to global cyber security threats. This inevitable transformation to highly connected systems thus necessitates effective security safeguards to be in place as any compromise or downtime of SCADA systems can have severe economic, safety and security ramifications. One way to ensure vital asset protection is to adopt a viewpoint similar to an attacker to determine weaknesses and loopholes in defences. Such mind sets help to identify and fix potential breaches before their exploitation. This paper surveys tools and techniques to uncover SCADA system vulnerabilities. A comprehensive review of the selected approaches is provided along with their applicability.
\end{abstract}

Index Terms - cyber defence, anomaly detection, attack tools, vulnerability, simulation, modelling, SCADA.

\section{INTRODUCTION}

$\mathrm{S}$ UPERVISORY Control and Data Acquisition (SCADA) systems are used to monitor and control critical national infrastructures such as smart grids, oil and gas, power generation and transmission, manufacturing, and transportation networks. They are also used to manage public utilities like buildings control, water, sewage, and traffic lights. The downtime or compromise of these systems can have disastrous consequences for the economy, public health and national security.

SCADA systems (Figure 1) are cyber physical systems with communication networks (wired and wireless) interfacing the monitoring and control system with the hardware and providing a large attack surface [1]. The architecture can be envisaged as four layers as shown in Fig 1. At the lowest level, field or slave devices (sensors, pumps, motors) provide an interface for control and monitoring of the physical process. At the next higher level, Remote Terminal Unit (RTU) and Programmable Logic Controllers (PLC) aggregate control (acting as master) for many field devices by passing commands and responses through the communications network to the SCADA server. PLC is a computer system running Ladder Logic for decision making to control the field devices. The operator monitors the process state through

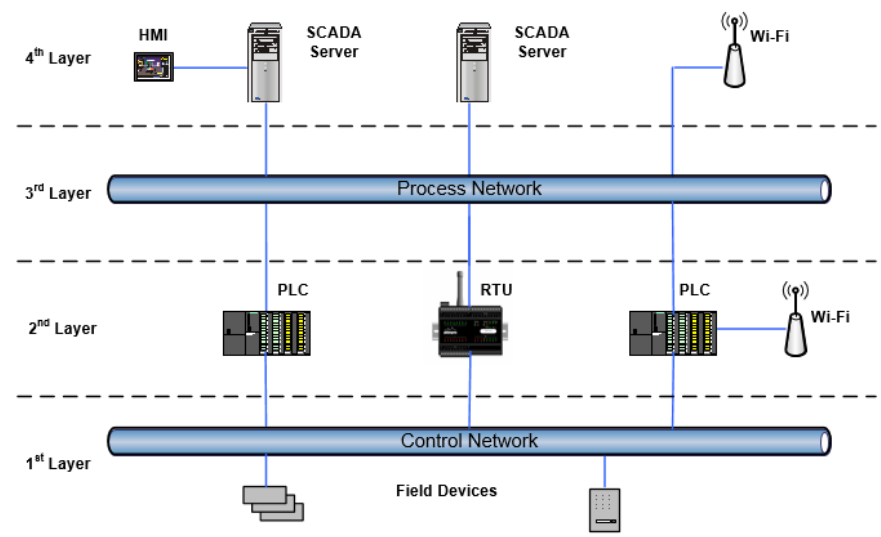

Fig. 1. A simplified layered architecture for typical SCADA system.

Human-machine Interface (HMI) and controls the process by activating commands as required [2]. A typical SCADA system could have multiple supervisory systems, PLCs, RTUs, HMIs, process and control instrumentation, sensors and actuator devices over a large geographical area, interconnected through a communications network.

The use and applications of SCADA systems has increased as a result of rising levels of industrial process automation, reduced cost of operation and growth in global economies. Growth is expected to increase in the use of SCADA systems and the investment is expected to reach up to $\$ 11.16$ billion by 2020 [3]. With the proliferation of the Internet of Things (IoT), SCADA sensor and actuator devices which are Internet connected SCADA systems are being transformed from a traditional on-site, stand-alone system to an Internet-connected remotely accessible system. An overview of challenges and security requirements for IoT is provided in [4]. A significant obstacle in IoT adoption is security aspects as it would be an attractive target for hackers [4], [5].

There are many benefits of Internet access including scalability, better communications protocols, efficiency, cost effectiveness, interoperability between components [6] and remote access, but SCADA systems were never designed with network connectivity and security [5], [7] in mind. The focus had always been on reliability rather than security, and protection had been ensured through isolation and obscurity [8], [9] by using proprietary standards. Since the 1990s the control systems are being integrated with computer networks [10] and also more and more Commercial-off-the-shelf (COTS) products are being used in SCADA systems [11]. SCADA server and user interfaces are now accessible over the Internet and cellular networks, providing many entry points 
for an attacker [8], [12]. Most SCADA communications protocols are just plain-text [13], [14] with no message authentication [15] making it easier for a man-in-the-middle (MITM) attack. TCP/IP protocols have their own vulnerabilities that can be exploited [5]. PLCs would treat code as legitimate as long as it has the correct syntax [16]. The threat landscape for SCADA systems has been broadened [8] by Internet and cellular network connectivity, bringing along open standards such as web technologies, which have known security loopholes making it very easy for an attacker to gain an in-depth knowledge of SCADA networks [17], [18]. The modern SCADA communications use a variety of communication media, such as $\mathrm{WiFi}$, cellular, and Bluetooth. Vulnerabilities in the communications protocols have been the main focus and target of cyber attacks. Failure to protect the SCADA infrastructure against the evolving threats of the changed connectivity landscape can have disastrous consequences. In the prevailing cyber security global environment, it is not a matter of if an attack of catastrophic proportion would happen, but rather when.

A Denial-of-Service (DoS) attack on a website can render a service unavailable, but similar attacks on SCADA systems can have potentially disastrous consequences [19] because of the fallout of the controlled process getting out of control. Stuxnet [16], June 2010, was the first malware designed to attack control systems and was the first attack of its kind that brought SCADA security vulnerabilities to prominence [19]. Prior to that although vulnerable, SCADA systems were not considered to be actively targeted. Malware, such as Flame (2012) that copied data, recorded Voice over Internet Protocol (VoIP) audio and intercepted network traffic [19]. Stuxnet (2010) and Duqu (2011) used USB devices to spread and attacked the PLCs by changing the Ladder Logic code [19]. Havex (2014) can reportedly infect the software downloads from the SCADA manufacturers' web sites [20]. An active group of attackers, Dragonfly [21], mainly target energy sectors through malware tools and infect targeted organisations using spam emails. These malware attacks highlight security weaknesses in SCADA system design [22]. Other attacks like Slammer at Davis-Besse nuclear plant [10] negate the illusion of security. The cyber attacks on SCADA systems have seen a $100 \%$ increase [23]. General technology awareness, widespread availability of free information, and the current global security situation of state and non-state elements with malicious intent, all combine to make launching such attacks easier and probable.

Countering the cyber attack is an emergent need to provide adequate safeguards against the cyber attacks by strengthening the defence. The general cyber security safeguards such as restricted physical access, cryptography, patch management, separation of corporate and production systems (through Demilitarized Zones (DMZ), Firewalls and Access Control Lists (ACLs), and activity logging are all applicable (Figure 2) but need to be viewed in conjunction with typical SCADA systems characteristics. Nonetheless these security measures are important as the corporate network could be the entry point for launching an attack on the SCADA network. Most of these security measures are not capable of defending SCADA systems from attacks against SCADA protocols [24]. For instance, SCADA characteristics make it difficult to apply existing cryptographic techniques, due to limited computational capability, low data rate, and the need for realtime response [17]. The confidentiality, integrity and availability (CIA) triad [25], applies to SCADA systems but with a changed order of priority as Availability, integrity and confidentiality (AIC), with availability being the most important. Agencies such as the National Institute of Standards and Technology (NIST), USA and European Network and Information Security Agency (ENISA), provide best practice documents for cyber security for SCADA systems in particular. Protection for telework devices is described in [26], Cyber security of SCADA systems in [27]. Guidelines for Patch management are provided in [28]. Protecting Industrial Control Systems (ICS) [2] has recommendations for Europe and member states, which identifies security challenges and recommends a common test bed for security testing. North American Electric Reliability Corporation (NERC) has released Critical Infrastructure Protection (CIP) documents. The industry regulations have started mandating the cyber security safeguards and this trend is likely to increase in the future.

Investigating the effect of an attack on an actual system is neither recommended due to the unintended consequences, nor feasible on a replicated system due to the cost and effort involved. Analysis methods and tools are very important to secure such systems [29]. Therefore SCADA cyber security researchers mostly rely on developments of simulation software and hardware to model SCADA attacks to analyse the system security. SCADA system security can be assessed by using vulnerability analysis through actively attacking a system which not only uncovers the vulnerabilities but can be

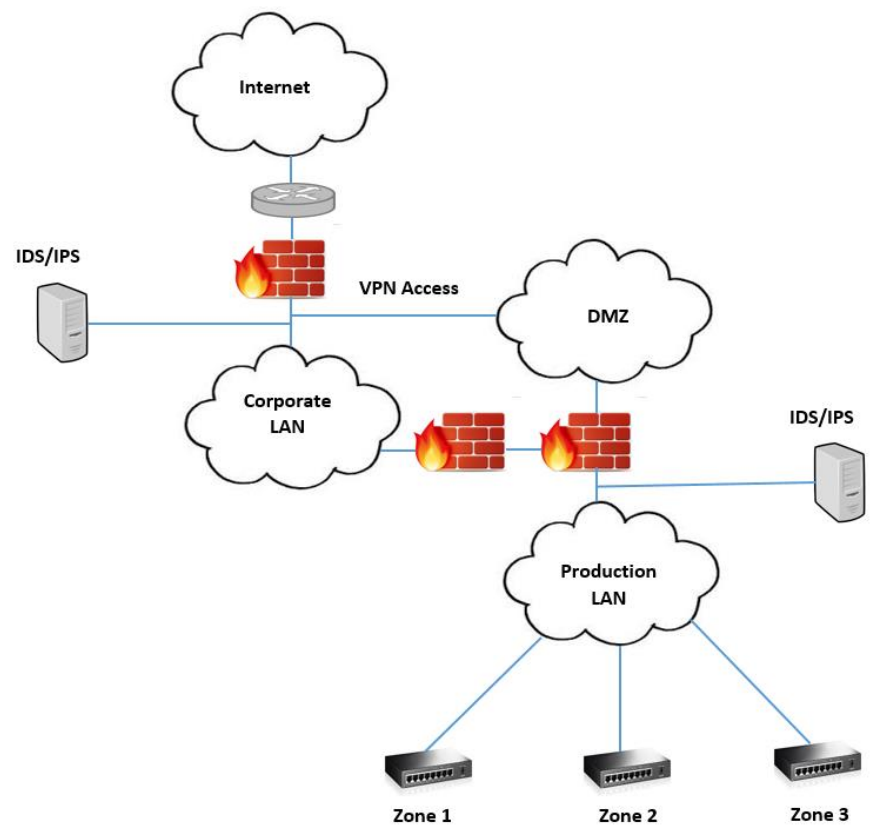

Fig. 2. DMZ with separation of trust zones. 
used to determine the system failure response, which helps to understand the system and provide necessary safeguards by fixing the vulnerabilities. Techniques such as penetration testing and vulnerability analysis can be considered inclusive in vulnerability assessment [30].

Generic Simulators for SCADA systems are described in [31] but the focus is not on cyber security. Smart Grid simulators [32] provide a useful reference for simulation tools but do not address SCADA or cyber security. Vulnerability assessment and analysis comprises of a spectrum of techniques from the simplest ones doing port scanning to those involving exploitation of vulnerabilities, as in an actual attack [27].

This paper provides a comprehensive survey of simulation, modelling and related techniques helpful for assessing the cyber-attack vulnerabilities of SCADA systems. In this paper we aim to cover the array of techniques for assessing SCADA vulnerabilities under simulation, modelling, tools and techniques as these are often employed by researchers for SCADA cyber security. This categorisation is purely with a view to better organise the research literature rather than a taxonomy. We also highlight recent technology innovations which can aid in minimizing the effect of cyber security risks.

The rest of the paper is organized into the following sections. Section II provides SCADA systems' characteristics and vulnerabilities. Section III covers the simulation and modelling techniques for identifying security weaknesses. Section IV describes other tools and techniques for evaluating defence. Section V provides conclusions, and Section VI discusses future research directions.

\section{SCADA SYSTEM CHARACTERISTICS AND VULNERABILITIES}

SCADA system (Figure 1) differs in characteristics from a conventional information technology (IT) system [8], [27]. SCADA systems have tighter constraints on reliability, latency and uptime that preclude some IT security measures [15]. SCADA are cyber physical systems, that is, cyber system (control and communications) and physical system (sensors, actuators) comprising a system of systems, interact as a cohesive and unified whole. The software commands manifest actions to modify physical processes. It is important to consider these differences when devising the protection strategies.

\section{A. Generic $O S$}

SCADA systems run over conventional operating systems (OS), thus inheriting vulnerabilities which can compromise the SCADA system [10]. The vulnerabilities of the operating systems are periodically announced by the vendors [33]. The patches are normally issued after vulnerabilities are discovered, but there could be a substantial time lag to release patches or the patches may not be applied in time. The patch for the vulnerability exploited by Stuxnet in 2010 became available in 2012 [28]. There is generally a time lag for patch application, for instance, Slammer infections occurred six months after the patch to fix the vulnerability had been released [10]. Similarly lack of user incentives [34] to apply patching enabled Code Red, a malware to infect 360,000 servers, although a security patch had been released earlier. In some cases, an attack comes before vulnerability is discovered and is termed as a Zero day attack.

\section{B. Legacy systems with long operational life}

The installation of SCADA systems is costly and timeconsuming and most systems remain in operation from eight to fifteen years [10]. A system may have devices from many different manufacturers using various standards or proprietary communications protocols [35]. This is sometimes well past the expected supported lifespan of the software and also hardware. Thus at times a system would comprise of legacy components and their associated vulnerabilities [29].

\section{Multiple Points of Entry and Failure}

A SCADA system is geographically spread over a large area starting at the sensors, in the field, to the user and control interface. Although SCADA servers may themselves be well protected against cyber attacks, however similar guarantees do not exist for field devices. The communication network, comprising of wireless Internet, cellular and Bluetooth provide multiple remote entry points which can be exploited by attackers. Wireless networks are especially vulnerable using freely available tools like Aircrack-NG that can sniff, test and even decrypt packets [36].

\section{Communication Protocols}

The low-level networking protocols used for industrial systems use simple plain-text messages based on a masterslave communications model. These lack security and encryption, as these were designed for isolated systems [13].

For example, Modbus protocol can be attacked as reported in [8], [37] with varying consequences [37]. Other recent protocols, such as Distributed Network Protocol 3.0 (DNP3) also have their vulnerabilities [5], [38], [39] and packets can also be analysed [36] through network sniffing tools to gain information and cause damage. Widely used protocols IEC 60870-5-101 and IEC 60870-5-104 lack application and data link layer security and have vulnerabilities that can be exploited [13]. With an understanding of the process and the protocol, an attacker can maliciously alter the process control by injecting valid control commands and responses with malicious intent [13], [22]. Attacks on protocol implementation [37] can cause failures resulting in possible exploits [8].

\section{E. Real-time and Complex Interactions}

SCADA systems monitor real-world processes under very tight timing and operational constraints. Time is critical for decision making, affecting a control system and vital process deviations, which must be accurately reflected and effectively managed. The stringent operational constraints (such as timing) of a SCADA system mean that it is more prone to fail in response to small deviations caused by an attacker. "Aurora Generator Test" [1], [10] in March 2007, simulated a remote cyber-attack resulting in destruction of a $\$ 1$ million dollar diesel-electric generator [40]. A patch application [25] or loss 
of time synchronization [1] may have unintended consequences detrimental to the prescribed operation. Application of a software update resulted in automatic shutdown of a nuclear plant [10]. Analysing and exploiting vulnerabilities may be complicated but unintelligent computer viruses and mere malfunctions in small devices can result in enormous unintended effects [10].

\section{F. Conflicting Priorities}

SCADA control and monitoring projects remain in continuous operation [41] for many decades after commissioning. This creates a dilemma for the administrators between ensuring adequate protection and sustained system operation. Application of software upgrades and patches may get postponed due to the desire to keep the system running without change to the execution environment [28]. Anti-virus and patches may result in undesirable consequences [10] or may also tend to slow down the communication and may interfere with normal functioning of the system.

The operational nature of these systems precludes post commissioning cyber security testing due to associated risks of jeopardising the controlled system.

\section{G. Social Engineering and Insider Attacks}

Social engineering attacks purporting to be from a known person or organization can be used to infiltrate a system. Often the cyber security is focused on an outsider's attack, which makes sense, but equally probable and dangerous is an attack originating from within the trusted network, through a deliberate or unintentional omission, or sabotage.

The attack in 2000 on a sewage control system in Queensland, Australia [10], [42] causing flooding with a million litres of sewage, was an act of a disgruntled employee. Stuxnet infiltrated the network [10], [16] mainly through USB sticks.

\section{H. Backdoors}

The Stuxnet [43] worm exploited system vulnerabilities to attack a PLC in Iran's uranium enrichment program in 2010. It exploited an administrative backdoor, which can be used to access a system remotely, and generally their availability on a system is known to system maker only. Such coded backdoor passwords which can be used to exploit a system remotely, are not uncommon [19], [44]. Such malpractice could also take place without the knowledge of a SCADA vendor, as increasingly the product is assembled from components manufactured from facilities across the globe [19].

\section{Integral Protection}

With cyber security awareness coming into prominence, SCADA manufacturers also provide and emphasize security in products. These features provide encryption and security features such as Kerberos and multiplexing proxy. Activating these in a project can make an intruder's task difficult. SCADA systems also provide other built-in mechanisms such as User Groups, Historian, Encryption and Redundant Servers.

\section{Simulation AND Modelling}

SCADA systems are not only complex but have many system interdependencies which makes it difficult for them to be tested for cyber defence. The production systems are required to provide a continuous and reliable service, and depending on the monitored process, even small delays are intolerable. As such the systems cannot be taken out of service for vulnerability checks, and also these are very costly and hard to duplicate.

Simulation and modelling techniques are useful to model and test complex systems. Development of realistic models help to create scenarios that do not yet exist or would be very costly to build. A model also makes it easier to quickly change parameters to suit another scenario or configuration.

Simulation and modelling techniques are used advantageously to evaluate and probe the defence of SCADA systems. A summary is provided in Tables I and II.

\section{A. Simulation Frameworks}

Simulation frameworks are needed to model all aspects of the SCADA system using simulators and emulators. Generally a network simulator such as OMNeT++ is used for network modelling and Simulink/MATLAB is used to simulate the process control. A framework in general also provides the facility to integrate the various simulators to realistically represent the system as a whole.

1) High Level Architecture (HLA)

HLA is a simulation integration platform designed by the Department of Defence (DoD) [45] that can be used to integrate simulators. This concept was chosen as no single simulation can meet all the requirements. An individual or a set of simulations can be applied across different uses, under the HLA federation concept. Federation means a set of interacting simulations, with each simulation termed as a federate. The federates must allow exchange of data through the Runtime Infrastructure (RTI).

HLA which is a co-simulation environment has been used by researchers to design simulations using OMNeT++ and MATLAB, for example.

Chabuksawar et al. [46] used Command and Control (C2) WindTunnel as a simulation framework (based on HLA) [47] to simulate a plant, its controller and the interconnecting network. The objective was to simulate network security attacks using this framework that requires domain-specific modelling language for defining integration models. The SCADA system was a simplified version of the Tennessee Eastman Control challenge problem [48]. DDoS attacks were simulated on the routers concluding a proof of concept implementation.

\section{2) SCADASiM}

An integrated framework for control system simulation, SCADASiM is presented by Mahoney and Gandhi in [9]. It can be modelled and simulated at different levels of abstractions commensurate with the problem at hand. The modelling notation is through Autonomous Component Architectures (ACA) that allows components to be modelled at simulation runtime. The authors proposed a new language 
Autonomous Component based policy Description Language for Anomaly monitoring in Control Systems) (ADACS) that was used for monitoring regulatory compliance.

3) SCADASim

Queiroz et al. [49] present a framework for building SCADA system simulations. Additionally it can be used to create malicious attacks against SCADA systems. The framework can be extended by SCADASim users to add their own protocols otherwise there are too many protocols. The framework is built on top of OMNeT++. Details of DoS and spoofing attack simulation are provided in the paper.

4) Co-simulation Framework

A co-simulation framework is proposed by Bytschkow et al. [50] using Common information model (CIM) as an intermediate model. It uses the approach of federation enabling both simulation and deriving possible impacts. The co-simulation framework is constructed using SCADA, CIM, GRIDLAB-D and AKKA.

\section{5) Emulation Framework}

A framework for emulation based security analysis using Emulab and Simulink is proposed by Genge et al. [6] that can be used to measure impact of attacks against both physical and cyber parts of systems. The authors' proposed framework extends Emulab to incorporate additional features required for cyber physical security analysis. The architecture comprises of a cyber layer, physical layer, and a cyber physical link layer. The authors provide a feature based, cost based and an experimental scenario-based in comparison to other frameworks reported in the literature and contend their approach to be better. The authors provide two case studies from the electrical and chemical domains. The first studies the effect of Stuxnet on a Boiling Water power plant showing that the proposed framework can be used to recreate a scenario with complex malware. The second studies the effect of network parameters on a cyber attack targeting a chemical process, showing that in cyber attacks where the attacker communicates with PLCs, the communications delays and packet losses have little effect.

6) Integration Framework

An integration framework has been proposed by Novak et al. [51] that advocates semantic and technical integration of simulation models into SCADA systems. The authors contend that simulations cannot be developed without access to online and historical data and thus propose a platform for integration of simulations and SCADA. It reduces design-time errors (for simulation) and improves re-configurability and reuse. Two case studies are provided for design of simulation models for passive houses, and an application allowing the management and execution of simulations.

7) Real-time monitoring, Anomaly detection, Impact analysis, and Mitigation strategies (RAIM)

The security SCADA framework proposed by Ten et al. [52] comprises of real-time monitoring, anomaly detection, impact analysis, and mitigation strategies (RAIM). Real-time monitoring can utilise the data for real-time control functions. Anomaly detection and impact analysis can be done through monitoring and correlating the system logs. The output is ranked as varying degrees of risks, based on which mitigation actions can be taken.

\section{B. Test Beds}

Test bed is a platform used to test systems or technologies where the actual system cannot be endangered by testing, due to unintended consequences, for example, checking the effects of patch application and response to malware. A test bed must capture the essence of the system under test for it to be useful. The facility can also be shared to save cost or share knowledge. Test bed creation is also recommended in [2]. Although some test beds have been developed by large organisations, generally the access is restricted to affiliated researchers only [53]. Unlike a simulation environment being fully contained in software, a test bed uses hardware, simulated and emulated devices. A survey of test beds in software and hardware is provided in [53].

Test beds could be realised [54] as simple simulation based (TrueTime), federated simulation (several dedicated simulation federates for plant, network etc. such as HLA) or emulation/implementation based (real hardware or emulator such as EmuLab).

\section{1) National SCADA Test Bed (NSTB)}

The Department of Energy, US, have established a National SCADA test bed [55] that aims to provide testing, research and training facilities to help improve the security of control systems. However free access to academia and industry is not available. Thus, many researchers have developed test beds to investigate some element of security.

2) TRUST

An experimental simulation test bed TRUST-SCADA [56] was aimed to assess and address vulnerabilities, and to provide an open-source design for a flexible test bed. DoD/HLA was chosen as the integration platform, for the plant model (Simulink/Stateflow), Network model using (OMNeT++, NS2, OPNET) and controller (Simulink/Stateflow).

The authors [54] have also proposed a test bed for SCADA vulnerabilities and validating security. The specific scenarios analysed were DoS attack on sensors, integrity attacks, and phishing attacks

\section{3) Live Virtual and Constructive (LVC) Test Bed}

Urias et al. [29] describe a hybrid test bed that can be used to perform cyber-physical security analysis. It was developed at Sandia National Laboratories to identify system level vulnerabilities, results of their exploitation, and approaches to eliminate it. Simulated network devices were represented using OPNET, enabling passing of simulated traffic to real devices. Virtual machines were used as hosts, servers and Cisco routers' emulation, and physical devices to which the simulated network traffic could be addressed. The experiments setup simulated the enterprise and control system network and provides analysis of cyber attacks against the business and control system network. The experiments investigated the effects of attacks on SCADA protocols (DNP and Modbus TCP) and how to mitigate such attacks using network security. 4) TrueTime

A simple test bed [57] has been proposed by Farooqui et al. using TrueTime (MATLAB/Simulink based simulator developed by Lund University) to simulate DoS attacks and its effects. The SCADA network is designed to control four different DC servo motors through a reference signal. The 
DoS attack scenarios covered attack on a PID controller and a specified actuator.

5) Research and Pedagogy

A test bed for SCADA cyber security research and pedagogy has been developed by Morris et al. [58]. It provides the facility to discover vulnerabilities, implications and classification of vulnerabilities by type, mitigations and validations. Developed at Mississippi State University, it models various industrial applications such as smart grid and gas pipeline, through hardware and software.

\section{6) TASSCS}

The OPNET test bed by Mallouhi et al. [5] is used to simulate networks, PowerWorld simulation system to simulate the functioning of power grid, and Autonomic Software Protection System (ASPS) for detection and protection against SCADA cyber security attacks. The attack detection is based on an anomaly based approach. It provides details of DNP, Modbus, and TCP/IP attacks through a training and detection phase.

\section{7) Power Station Simulation}

The test bed proposed by Hahn et al. [59] consists of power station simulation, substation automation and SCADA system, and uses scenarios based on anomaly detection. It is based on real-time monitoring, anomaly detection, impact analysis, and mitigation strategies (RAIM). The test bed uses ICCP, DNP 3.0 over TCP/IP, and OPC communication protocols.

\section{8) Power Control System}

A test bed for a simulated power control systems is reported by Dondossola et al. [60] for collection of data through controlled experiments on a power system test bed, and activities for using the collected data for analysis and risk assessment frameworks. Cyber threats such as DoS and false injection attacks were investigated. The authors also gathered statistics based on message delays, number of lost messages, and time to failure to UDP flooding attacks.

\section{9) Common Open Research Emulator (CORE)}

Tesfahun and Bhaskari [61] propose a scalable and reconfigurable virtual SCADA security test bed for developing and evaluating security solutions. The authors provide a labelled dataset for other researchers. It is based on Common Open Research Emulator (CORE) for simulating SCADA networks. It is possible to launch multiple attacks simultaneously and benchmark datasets can also be generated. CORE can be connected to real world networks, with the Python module to customise network emulation. The researchers represented SCADA devices by a virtual node in CORE. DDoS and False Data Injection attack were simulated.

\section{0) Software Defined Networking (SDN)}

SDN makes it possible to dynamically reconfigure an IP network. Dong et al. [1] explore the use of SDN techniques for enhancing the protection against cyber attacks. The authors propose a co-simulation test bed comprising Mininet (to emulate smart grid communications), and PowerWorld (to simulate physical aspects of power systems). The test bed has Bro-based IDS to analyse the DNP3 traces and provide results for SDN countermeasures. Three use cases were considered to demonstrate the SDN potential for strengthening the resilience.

\section{1) SCADA Virtual Test Environment}

A test environment is proposed by Boldea [62] to assess the security of SCADA systems, and the use of virtual systems to emulate the real systems, and used GNS3 for network components and Virtual Box for software virtualisation. The SCADA test bed used the free SCADA 2 software with a Designer and Runtime tool to simulate DDoS attacks.

\section{Simulating SCADA Attacks}

Simulating SCADA attacks makes it possible to explore the cyber defence of the system under investigation. The results can then be used to strengthen the defence.

1) Malware Attacks

Malware or malicious software poses a serious threat to SCADA systems. The vulnerabilities present in the IT and communications systems can be exploited by viruses and malware, hence making SCADA systems vulnerable to such attacks as reported by Fovinoa et al. [24].

A malware attack simulator for testing SCADA system under controlled environments, Mobile Agent Malware Simulator MAlSim has been proposed by Leszczyna et al. [63]. The toolkit provides the facility to implement various types of malware. The aim was to provide security assessment based on simulated attacks. It can be used to simulate wellknown malware such as Code Red, SQL Slammer. A malware template is comprised of a MAlsim agent with its behavioural and migration patterns.

MAlsim was used by Fovinoa et al. [24] to investigate malware attacks on a SCADA system on a power plant test bed comprising of a process network, field network, intranet, data exchange network, external network and observer network. The code for Code Red, Nimda, Slammer and Scalper was obtained and injected into the process network to activate these malwares. The malwares infected the machines but did not lead to system failures. The authors also provide results for a Modbus DoS and network attack.

Ciancamerla et al. [64] provide results for a malware injection on an electric grid.

2) Network Attacks

Chabuksawar et al. [46] used a simulator that uses C2WindTunnel. The paper emphasizes co-simulation of controller and plant dynamics in Simulink/MATLAB and network architecture and behaviour in a network simulator like OMNeT++ [24].

NETA is a framework for the simulation of communication networks attacks. Network Attacks (NETA) [65] is based on OMNeT++ and provides a framework for simulating attacks in heterogeneous networks.

\section{3) Communication Protocol Attacks}

There are hundreds of communications protocols in use for SCADA communications. Jin et al. [39] provide modelling of buffer flooding attack on DNP3 protocol. A simple flooding attack fills the event buffer in the data aggregator so that the critical alerts from legitimate devices cannot be buffered which impacts the control station's situational awareness. The behaviour is analysed through a simulation model [39]. Moya 
et al. [66] describe a Grey Hole attack against a SCADA substation using DNP3.0.

Fovino et al. [67] provided a filtering system based on state analysis for securing SCADA protocols, Modbus and DNP3. The aim of the study was to detect attacks where a set of licit commands on execution can disrupt a SCADA system while in particular states. A firewall does not guarantee complete protection to SCADA systems, as it operates on a signature-based approach. Thus a firewall needs the system state and the set of unwanted states. In order to check whether the system is proceeding to a critical state from which the distance from critical states can be calculated. The proposed method was validated on a prototype system.

A ' $C$ ' language graph based implementation by Genge and Siaterlis [22] for network segmentation separates control hardware regulating input flows from output flows of the industrial process for SCADA resilience. The human expert is needed to construct a directed graph where vertices are process units and edges are product flows, the segmentation is performed through a heuristic algorithm. The methodology was applied on the Tennessee-Eastman chemical process using two attack scenarios on PLCs using Modbus protocol and the results show that it can be used for defence against Stuxnet like attacks.

A graph theory analysis for IEEE 118 bus system is presented by Srivastava et al.[40].

\section{4) Denial-of-Service/MITM}

This has been the most well studied type of attack as it is easy to implement and launch. A malicious agent can flood a specific device through protocol exploitation, resulting in bandwidth saturation that renders the service unavailable as described by Ciancamerla et al. [64]. SCADA system vulnerability analysis through DDoS attack is presented by Petrovic and Stojanovic [74]. The simulation considers a corporate and SCADA network. A DoS attack on an actual SCADA system of a medium voltage electrical grid is provided in [64]. Malware attack results for DoS for Modbus protocol is provided in [24].

The wireless packets are easy to exploit because the intruder does not have to be physically connected to the network (as in wired) to access the network traffic. Xie et al. [68] have proposed a simulation platform based on radio modem for analysing radio modem security. Radio modems are typically used for long range communications to connect PLC, RTU etc. but often the data is sent in plain text that can be exploited. The paper explored four types of attacks, that is, communication jam, data eavesdropping data tamper and eavesdropping, and DoS attack.

MITM attacks on IEC 60870-5-104 SCADA networks are described by Maynard et al. [76]. Details of the protocol packet payload are provided. MITM attacks will follow the stages of detection (to identify targets), capture (data collection), and finally attack. The experiments cover relay and MITM attacks and, attackers with varying degrees of experience can compromise the system by hiding fault condition from a SCADA server.

5) False Data Injection (FDI)

In False data injection attack the stored or transmitted data from RTUs, control centre or communications infrastructure is modified with a malicious intent [77].
Hug and Giampapa [77] considered the FDI attack on a SCADA system for a power grid for ac state estimation. Through simulation using IEEE 57 bus system, details are provided for a number of measurements that the attacker needs to alter, to stay hidden. If the attacker has knowledge of the system data then the attack will not be noticed through the ac state estimation. FDI were also investigated in [60].

6) False Sequential Logic Attacks

Li et al. [79] proposed a false sequential logic attack on a SCADA system. An informed attacker can alter the sequential logic of control to disrupt the physical process before the intrusion is detected. The sequential logic of the physical process is modelled as finite state machine (FSM). Traditional IDS will not be able to detect an intrusion as it is based on licit commands, demonstrated for a three tank system. To detect the proposed attack there is a need for sequential logic featurebased IDS to continuously monitor the control sequence.

\section{7) Integrity Attacks}

An attacker can gain access to the sensors and/or actuators and modify the software to launch a coordinated attack as reported by Mo et al. [80]. Data integrity attacks wherein the sensor or control signals are manipulated can have severe consequences as the operator could be misled into taking wrong actions. These attacks are more difficult to overcome as their onset is not as obvious as DoS attacks. In [80] the authors focused on techniques for integrity attack detection and describe an analytic approach verified through simulation for detecting replay attacks on sensors. It assumes that the attacker has capability to read sensor inputs and capability to inject input.

Such an attack however would require knowledge of the system as described by Sridhar and Manimaran [81]. In [81] an integrity attack is simulated on an Automatic Gain Control (AGC) loop that keeps both tie-line flow and frequency deviation values correct. Simulation is performed on a twoarea system, and verifies that the system can be led to an unhealthy state by an attacker manipulating values intelligently.

Unsupervised anomaly detection for integrity attacks on SCADA systems is described in [11].

8) Real-time and Simulation Monitor

A methodology to ensure SCADA availability through a real-time monitor and a simulation monitor is proposed by Shen et al. [82]. The real-time monitor, monitors states and events and based on that, estimates if there are faults or risks. The simulation monitor simulates control commands, monitors and predicts the results of those commands and estimates whether the commands are dangerous or not. The methodology is then tested on a simulated water treatment system.

\section{Mathematical Modelling}

Modelling techniques provide a reliable and formal mechanism for validating a system under attack. Linear dynamical models [30] are used to model the behaviour of control systems. A model for a web robot network (botnet) is proposed by Brand et al. [83], which can be used to attack the system in different ways. Botnets can bring down a server through a DDoS attack from many compromised machines as investigated by Baecher et al. [84]. 
Backhaus et al. [12] describe a game theory model to outline a scenario where the attacker, after gaining access to the system will interact through its control system with the system operator, and the outcome of these machine-machine interactions will be governed by the design of the physical and control systems. Considering a simple model, the interactions of the attacker and defender are explored and the outcome is estimated. Extensions to real world complex problems would increase the computational requirements exponentially.

Yang et al. [85] proposed Factor Neural Network (FNN) to study the security problem in SCADA through developing a FNN-based security defence architecture model. The attack and defence of SCADA is taken as online digital intelligent antagonising process and all reasoning, judgement and thinking is abstracted into corresponding network attacks and defence knowledge system. The proposed model needs further research into SCADA network attack simulation.

Cardenas et al. [75] use a mathematical formulation to detect and survive attacks in specific research problems. The physical system is modelled as a linear dynamical system.

Testing complex SCADA systems is challenging, Süß et al. [86] propose the use of Modelica and Eclipse Modeling framework. Modelica is a mathematical modelling language for complex physical systems and offers Ecore, the metalanguage of the Eclipse framework. The focus is on an integrated unified model driven development environment.

\section{E. Probabilistic Modelling}

Queiroz et al. [87] propose a survivability model based on Bayesian networks, taking into account the type of protocol communication. The focus is on system survivability despite attacks. The simulated system consisted of fibre networks modelled using SCADASim [62] to simulate and test the model. Such techniques are very useful as the complex interaction between system components can be easily validated. A Bayesian attack graph model is proposed Zhang et al. [88].

\section{F. Risk Modelling and Assessment}

A review of risk assessment techniques is provided by Cherdantseva et al. [89]. Risk management reduces the likelihood of cyber attacks disrupting SCADA and in the event of a successful attack reducing the severity of the consequences as described by Henry and Haimes [90].

An integrated methodology for managing the risk of cyber attacks is reported in [91]. Minimax envelopes are developed for dynamic multi objective models to address scenario uncertainty, due to different attacker motives and points of access.

A Network Security Risk Model (NSRM) for cyber risk analysis of the control system is proposed in [90]. The model is applied on an example system of a simplified crude oil pipeline pump station. NSRM is an attack model with a directed graph, where nodes represent process components and edges are the linkages from one process component to another. The model defines the state space where transitions take place with transition probabilities in response to attacker's actions.
A survey of available tools for SCADA risk assessment is provided by Ralston et al. [92]. It mainly covers probabilistic risk assessment to estimate the risk from SCADA systems.

A network vulnerability analysis using attack graphs is provided by Phillips and Swiler [94]. The attack paths and their probabilities could be identified and vulnerable system components can also be identified. In attack graphs, each node represents a possible attack state and each edge represents a success probability. The inputs to the system are configuration files, attacker profiles, and attack templates. An example is provided for generation and analysis of graph.

Attack-Trees were first described by Schneier [69] and are a widely used technology for risk assessment of safety-critical systems. The attack goal is modelled as the root of the tree and various possible ways of accomplishing the goal are the leaves. These make it easier to identify the more probable causes and make predictions. Attack trees visually describe the possible attack paths and can be used for risk assessment as described by Bouchti and Haqiq [70].

Moore et al. [71] provide guidance on documenting security attacks in a reusable form through an example. The practicality of an attack tree for a real-world system is governed by re-using an attack pattern. Through the chosen example of an enterprise, the authors describe the documentation of security attacks in a reusable form. Thus it provides a means to organize historical attack data for later analysis.

The attack tree methodology was used by Byres et al. [72] to model cyber attacks on SCADA systems. The authors provide some examples of risk analysis for attackers' goals of gaining access to the SCADA system, identifying Modbus device and compromising the master, and highlight the security issues. The authors describe their methodology through identifying eleven attackers' goals which were elaborated for their technical difficulty, probability of detection, and underlying critical vulnerabilities. They conclude that all the attack avenues depend on an attacker getting network access. The authors point to more rigorous work that is required for the techniques to be usefully employed.

Ten et al. [73] used attack trees for vulnerability assessment of SCADA systems. The paper considers an analytical model to measure vulnerabilities of a control centre. The methodology used vulnerability index as likelihood that an attack tree or leaf will be compromised.

Bouchti et al. [70] extended the attack trees with new modelling constructs and analysis approaches to propose Colored Petri Net (CoPNet) to model intrusion. Petri Net is a mathematical modelling language that can be used as a visual communication tool. Based on the mapping rules, a CoPNet model can be built from Attack trees. CoPNet can model both defences and attacks, unlike an attack tree that can only model attacks. The proposed method is applied to a 3 bus power grid and its SCADA network. The model provides better modelling compared to attack trees but has a more complicated form.

Attack trees have been used by Ten et al. [52] for intrusion modelling. The study is focused on the ports and passwords on control network computers. The vulnerabilities were depicted as a risk table. The hardening through administrative passwords was also tested. 
Bayesian attack graph models are applied by Zhang et al. [88] for power system attack scenarios of breaker trips through IEDs. A mean time-to-compromise (MTTC) model is used to estimate time for successful intrusion of cyber components. Bayesian networks model attack graphs using probabilities. Two attack graph models are considered, first is the attack graph of vulnerabilities, and the second estimates successful intrusion on communications links. The reliability analysis is provided for the attacks considered.

\section{TOOLS AND TECHNIQUES}

In this Section, we describe the tools that can be used either for gathering more information about an intended target system or those which can actively attack a system with or without such analysis. A summary is provided in Table III.

\section{A. Scanning Tools}

Any information about network addresses or open ports of a potential target can help the attacker to develop an attack methodology. By knowing which ports are open and listening, it is easy to infer about the running programs and then devising an exploit or attack methodology. If the attacker has access to the network, then through freely available tools it is possible to gather information about a system or to actively target it. In general the more information that gets collected the higher will be the damage caused [13]. Using similar tools as available to a hacker, can help to determine the weaknesses of the system and to provide a timely fix.

Nmap [95] is a freely downloadable scanning tool that can be used to gather information about a single machine or the whole network. It can provide information about the open ports, services being run and the operating system, and even the firewalls in use, as well as other characteristics. All of this provides valuable information to an attacker to plan the attack. Port scanning is often done before the penetration testing. Traffic to an open port would legitimately pass through a firewall and may be used to determine the Trojan or other malicious code running on a machine. However, Nmap can be run from one of the machines in the network which may be difficult for an intruder.

In contrast to a wired network, packets on a wireless network are easy to intercept because the intruder could intercept packets just by being in the range. There are tools such as Aircrack-NG that let packets to be captured.

\section{B. Penetration Testing}

Tools such as metasploit [96] can be use for penetration testing. Sploitware [97] which is a framework designed specifically for penetration testing of SCADA systems can be used to check for SCADA vulnerabilities.

\section{Machine Learning}

Machine learning techniques are mostly based on statistics and can analyse the process data to isolate anomalous data that signal malicious behaviour. Thus making automated machine learning techniques more appropriate and efficient compared to human analysts [98].

Almalawi et al. [11] proposed an unsupervised anomaly based detection scheme for a water distribution system to detect inconsistent states using k-nearest neighbours (KNN) and clustering using k-means. The inconsistencies could be either inconsistent network traffic pattern or SCADA data [11]. Simulated and real data sets are used to simulate MITM attacks on Modbus/TCP. The authors show their scheme to perform better than supervised and semi-supervised schemes.

Machine learning techniques have been applied to telecommunications; [98] proposed one class SVM for automated anomaly detection from SCADA telecommunications data.

Torrisi et al. [78] propose SVM based traffic analysis using message direction and timing information to protect against Grey Hole attacks. Unlike other work that is focused on identifying the different protocols in an encrypted tunnel, the authors consider an attack classifying messages that belong to the same application layer protocol, DNP3, and investigate the ability to cause interference in SCADA monitoring. In a grey hole attack, as the solicited responses from the master are let through and the unsolicited messages are dropped, the master would still not be aware of the message drop and thus the attacker can remain undetected. The message drop would result in the operator observation to be off by about $10-20 \%$. Such attacks could be mitigated through use of TCP as the sequence numbering works in both directions and loss would be detected or by modifying the DNP3.0 protocol to use related sequence numbers for both unsolicited and solicited messages.

SVM techniques were used in [99] to identify malware and demonstrate use of an 'eigenvector' pre-filter to remove irrelevant features from the dataset.

Nader et al. [100] propose to detect malicious intrusions through machine learning after they have bypassed IDS. The paper investigates $1_{p}$-norms in Radial Basis Function (RBF) kernels for intrusion detection using one class classification techniques of support vector data description (SVDD) and the Kernel Principal Component Analysis (KPCA). The selected algorithms are applied on the gas pipeline test bed and compared to other selected methods, was faster, had higher error detection rates, and lowest false alarm rates. Application on a water treatment dataset gave better results for KPCA compared to SVDD.

A cloud based data analysis system for Los Angeles Smart Grid Project is described by Simmhan et al. [101]. It was based on Floe data flow framework which is hosted elastically on VMs and is supported by major cloud providers. The work demonstrates value of cloud computing and data analytics for smart grids but provides insights for mining similar data for just SCADA systems. Some principles for smart grid analysis are provided in [102] by Accenture.

\section{Network Intrusion Detection Systems (IDS)}

IDS work by inspecting the network traffic and mainly comprise of two approaches: signature based and anomaly based. The signature detection matches traffic to a known misuse pattern, while the anomaly detection works on the normalities in the observed data and can detect unknown attacks [14], [15].

A review of IDS schemes and a decentralised multi agent scheme is proposed by MacDermott et al. [103]. Digital Bond Quickdraw project [104] releases IDS signatures for DNP3, 
EtherNet/IP and Modbus/TCP that can be used to identify possible attacks [14].

A rule-based IDS is proposed by Yang et al. [14] for IEC 60870-5-104 protocol which is used for basic telecontrol tasks but the messages are in plain text and it also has inherent TCP/IP issues. The authors use Internet Traffic and Content Analysis (ITACA) tool for traffic sniffing. The proposed signature and model based approaches were validated by capturing normal traffic followed by abnormal packets, and effectively identified all abnormal data for the given rules and dataset.

This work has been extended by the authors of [14] for IEC/ 60870-5-104 protocols by deriving stateful protocol analysis approach [105]. Stateful analysis compares predetermined acceptable protocol behaviours against observed activities to detect deviations or intrusions. A detection state machine is proposed and applied for stateful IDS for SACDA systems.

Similar to attack tools, there are freely available tools that make it possible to detect and prevent an intrusion. A guide [106] to intrusion detection and protection is available by NIST.

Malicious users understand signature-based technologies and can craft malware that can elude such systems and remain undetected, as described by Winn et al. [107].

Some work has been reported based on machine learning techniques. The communications data sets from SCADA are analysed by Jiang and Yasakethu [98] with one class SVM to cluster the anomalies and generate an alarm based on perceived severity.

Oman and Phillips [35] described an implementation of a customised IDS and event monitoring system. The system can assist operators to identify erroneous or malicious settings and activities in the system.

The inadequacy of rule based approaches with reference to firewalls is elaborated in [67] by stating that for control systems, even a sequence of licit commands can lead the system to an unsafe state.

IDS based on critical state analysis in a power plant are proposed by Carcano et al. [108]. The authors contend that the system critical states, as a result of cyber attacks or system faults, can be segregated based on IDS that is aware of such critical system states, from known or unknown attacks. The authors develop a new Industrial State Modelling Language (ISML) and use it to define states. By monitoring the system states a critical state can be detected before it occurs by monitoring the distance form a critical state. The proposed scheme can also detect zero day attacks as it is based on system states from known to critical.

Kirsch et al. [41] describe what they term as a first survivable SCADA system using replication of SCADA master that continues with minimal degradation during cyber attacks. The system runs several copies of SCADA master thus the application acts as its own firewall and does not require prior knowledge of attack signatures. The replication protocol assumes that some of the replicas are compromised. The authors propose a state machine approach where all replicas start in the same initial state and cooperate to execute an event that ensures all replicas proceed through the same state sequence. Prime client library is used to link RTUs and
HMI to SCADA master. A polling and scalability scenario were used to validate the proposed system.

Snort [109] is a free tool for intrusion detection that can analyse traffic, packet logging, and protocol analysis. OSSEC [110] is another open source tool for intrusion detection. An early detection of an intrusion can help to contain its effect and potential damage [98], thus making such techniques extremely useful.

\section{E. Intrusion Prevention Systems (IPS)}

An IPS performs the intrusion detection and additionally also attempts to prevent/stop certain incidents [103]. An IPS monitors the network for any malicious activity and also attempts at stopping the intrusion, and raises an alert. Snort [109] can also perform intrusion prevention.

There is little research reported on IPS unlike IDS which is comparatively heavily researched.

\section{F. Honey pots (also conpot)}

Honey pots are computer systems deployed as decoys to attract hackers to attack them and thus record the attackers' actions. Thus sources and intentions of the attackers are obtained without exposing an actual system to exploitation risk. They provide knowledge about the tactics and techniques employed by the malicious users [107] and also about the origin of such attack.

The implementation could be a low-interaction honeypot (LIH) that offers limited services or high-interaction honeypot (HIH) that implements a complete system [19], [84]. Honeyd and GenIII honeynet are examples of a LIH and $\mathrm{HIH}$ respectively [84]. For details of different honeypot based tools and their relative merits please see [84].

Honeypot should mimic a device (such as PLC) as part of a larger system to be of interest to the attackers as described by Winn et al. [107]. Honeyd is a cost effective solution to deploy a realistic honeypot. During pilot studies it was used to advertise 75 PLC in [107]. Disso et al. [19] used honeynet Honeywall CDROM in a virtual machine as a honey pot. A PLC (low interaction) was emulated using HoneyD, and another PLC as high interaction.

Although mimicking an industrial system is complex, the open source honeyd [111] makes it easier. The attack traces can be stored and analysed to determine the sources of attacks. The information about the potential people interested in acquiring information, hacking, and frequent visits can help to bolster up the defence. On identifying a honey pot, the hackers may employ anti-honeypoting techniques [107].

A study to identify and group the traces left on honeypots to the botnets' originating machines is described by Pham and Dacier [112] that enables identifying new botnets. The traces are represented as time series that could be arranged based on the country of origin of a source. The time series can then be correlated to detect attack events. The attack events then help to identify attacks from the same botnet or a group of botnets.

A large scale collection of malware [84] can help design counter-strategies such as network and host IDS. Baecher et al. introduce Nepenthes as a platform to deploy honeypots as vulnerability modules. It's a scalable solution to emulate different operating systems and authors report experiments by emulating more than 16,000 different IP addresses on a single 
physical machine. Nepenthes is effective at detecting zero day attacks but is capable of collecting malware that is autonomously spreading. Their system collected 15,500 unique binaries over a four months period, and analysing them with different anti-virus systems detected $80-90 \%$ as malicious, that is different anti-virus engines are missing a significant percentage of malware.

Brand et al. [83] describe a malware rebirthing botnet that can be used to collect malware and rebirth it with new signatures to launch an orchestrated attack and avoid detection by AV software.

Viruses can be countered by propagating the immunization agent as an epidemic as proposed by Goldenberg et al. [113]. The authors propose using the honey pot architecture for early virus discovery and fast antivirus dissemination. They provide a concrete example of an email network.

\section{G. Security Information and Event Management (SIEM)}

SIEM works by aggregating the information from the selected tools to a central repository for real-time trend analysis. An open-source product is OSSIM [93], [114]. Mahboob and Zubairi [93] proposed OSSIM (by AlienVault as above), that is an open source Linux based Security information and event management (SIEM) system for SCADA security by configuring OSSIM. OSSIM can bring together several security tools such as Open source security (OSSEC) and a GUI. OSSIM can correlate events from different sources such as firewalls, anomaly detectors, IDS/IPS, and network switches and combine these with known vulnerabilities. The authors used a PLC (as VM), Honeynet and Honeywall VM for GUI. Snort is used for IDS and OpenVAS for vulnerability scanning. Based on the results mitigation actions (patches) can be taken and the scanning can be performed again. OSSIM assigns a risk value to each event and has many other correlation features not fully explored by the authors.

\section{H. Ethical or White-hat Hacking}

The term white-hat hacking means to perform the same actions as that of a real or black-hat hacker to determine the security weaknesses in a system with the intention to fix them before exploitation. Encouragement through recognition and rewards for finding and reporting vulnerabilities will bring such skills to prominence and help protect systems from malicious black-hat hackers. At a more formal level [115] describes the certification of the cyber security skills

\section{Forensic Science}

It may be useful to do post attack analysis for protecting the systems as investigated by Erol-Kantarci and Mouftah [7] against similar future attacks. It is a new research area for SCADA with similarities to digital forensic in other areas. Valuable information can be gleaned from events preceding an attack. However as pointed by the authors there are some challenges such as live analysis and issues like privacy of data etc. that need to be overcome.

Forensics [112] has also been applied to honey pot traces to identify new botnets originating from the same source machines or countries.

\section{CONCLUSION}

SCADA systems have gained prominence and widespread use beyond the traditional applications for highly critical systems such as power generation and transportation systems. Internet connectivity has changed the threat landscape and the recent interest and ability to monitor and control processes over the mobile network means even more diverse entry points. Thus effective strategies are required that can provide adequate protection against cyber-security threats and attacks. Perhaps the most important transformation needed is a different threat perception for SCADA systems.

Current strategies such as simulation, modelling and other approaches reported in the research literature for determining the efficacy of a system against a cyber-attack have been reviewed in this paper. These techniques can be used to uncover the system vulnerabilities by determining the degree of protection against a possible attack. This helps the system developers and providers to assess their systems before commissioning, and system users/clients to be aware of security provisions and compliance to regulatory requirements.

In view of the fast changing cyber threat landscape, adoption of security techniques will be offset by corresponding new threats being evolved. Hence there would always be a need for continuous evaluation and evolution of cyber defence practises to match the corresponding threats. The guidelines provided by the agencies [2], [106], [122] are steps in the right direction to lay down industry's best practices. One of the promising techniques in this category is penetration testing, especially by third party that can help to expose hidden vulnerabilities [17] and implement corrective action enabling system validation and remedy of any security weaknesses.

There are other promising techniques, such as simulation, modelling, and security assessment and honey pots. This coupled with the desire of the SCADA vendors to provide integration with commercial database systems, will make it possible for real time data analytics to identify a threat vector before it strikes. The selected techniques are important for the system developers to confirm adherence to security policies and certify a degree of protection against threats.

\section{FUTURE RESEARCH DIRECTIONS}

Recent technological developments in communications and networking have revolutionised the control and process networks making it much easier to access the data remotely and conveniently. The current research for cyber security protection has many promising techniques.

The emerging techniques such as SDN (highlight reconfigurability) and virtualization platform provides many benefits such as copying, restoring, deleting and backing-up virtual machines (VMs) on the fly. High Availability and Vmotion which enable continued operation of a virtual machine during migration. The virtualization platform provides many benefits such as isolation, snapshots, migration and restoring of virtual machines. Virtualised deployments are thus easier to protect compared to physical servers. Through 
update manager the vital updates can be automatically downloaded and applied.

Cloud computing is still a new technology for SCADA [116]. The control and monitoring industry has not yet fully embraced cloud computing because it is different to conventional IT systems. With further increase in network speeds, reliability and storage technologies; SCADA servers could be hosted on the cloud infrastructure. The advantages would be an easy enforcement of security standards, data analytics and disaster recovery. A technology similar to cloud that is gradually being adopted by the control industry is virtualisation. With a private cloud [101] or virtual infrastructure an organization can have the benefits of on-site SCADA deployment and the benefits of disaster recovery, migration, and high availability. This also ensures keeping the data latency to a minimum.

In future, more open communications standards for SCADA systems are expected to be adopted reversing the trend where most of the products were closed and proprietary. There are open source projects such as, OpenSCADA [117]. Although debatable [118] whether better protection is offered by a closed system by 'obscurity' of its implementation, or an open system, where the source is a public domain with a possibility for misuse by implementing a targeted attack. In the case of open systems, the user community can help by providing fixes both before and after vulnerability gets exploited through an attack. Such fixes could be quicker as there are more people using the system with full knowledge with more likelihood to uncover a potential threat.

The OPC UA (Unified Architecture) is an open industrial [120], [121] Machine-to-Machine communication protocol that replaced OPC DA. OPC UA is a set of 9 standards, with one devoted to security. The general concept is to simplify the SCADA communication interface by providing a common medium of communication. [119] used OPC communication from SCADA systems to collect system data for modelling a water distribution network. The data from the OPC server could similarly be used to investigate real-time cyber security issues by applying data analysis techniques.

Machine learning and data analytics have now advanced and are increasingly being used in new application domains. The large data generated [61] in a smart system can be used to extract information through data analytics for effective management. Machine learning techniques can be very useful for implementing strategies using an anomaly based unsupervised detection [11] approach for detecting attacks on SCADA systems. Future deployments of SCADA projects would see tighter integration between the process data and machine learning based data analysis engines observing historical data for anomalous behaviour to thwart cyber security breaches.

With industry regulations mandating cyber security for the SCADA systems, vendors will provide more built-in security features in their systems against cyber-attacks. For example, features such as multiplexing proxies

There is also a lot of ongoing work to improve the communications protocols [15] to provide better protection against attackers. For example, security was added to DNP3 protocol by creating its extension called DNPSec [24]. These developments are to be seen in the context of emerging IoT or smart devices which are now common in SCADA networks. There are both benefits and pitfalls to their use with the security as the main hurdle to their widespread adoption. IoT with its unique IPv6 addresses is both an opportunity and challenge for cyber security.

In future, there will be a need for lot more collaboration [42] between researchers, academics, vendors, developers, and government agencies to design foolproof solutions through integrated and cohesive efforts to meet the security challenges.

\section{ACKNOWLEDGMENT}

The authors gratefully acknowledge funding support from Innovate UK for sponsoring the KTP project at London South Bank University, in collaboration with Firstco Ltd.

\section{REFERENCES}

[1] X. Dong, H. Lin, R. Tan, R. K. Iyer, and Z. Kalbarczyk, "Softwaredefined networking for smart grid resilience: Opportunities and challenges," in Proc. of the 1st ACM Workshop on Cyber-Physical System Security, New York, NY, USA, 2015, pp. 61-68.

[2] Protecting Industrial Control Systems, Recommendations for Europe and Member States, ENISA, 2011.

[3] SCADA growth, [online]. Available: http://www.marketsandmarkets.com/PressReleases/supervisory-controldata-acquisition.asp

[4] S. Li, T. Tryfonas, and H. Li, "The internet of things: a security point of view", Internet Research, vol. 26, no. 2, 2016, pp. 337-359.

[5] M. Mallouhi, Y. Al-Nashif, D. Cox, T. Chadaga, S. Hariri "A test bed for analyzing security of SCADA control systems (TASSCS)," IEEE PES Innovative Smart Grid Technologies (ISGT), Jan 2011, pp. 1-7.

[6] B. Genge, C. Siaterlis, I. N. Fovino, and M. Masera, "A cyber-physical experimentation environment for the security analysis of networked industrial control systems," Computers \& Electrical Engineering, vol. 38, no. 5, Sep 2012, pp. 1146-1161.

[7] M. Erol-Kantarci, and H. T. Mouftah, "Smart grid forensic science: applications, challenges, and open issues," IEEE Communications magazine, vol. 51, no. 1, Jan 2013, pp. 68-74.

[8] B. Zhu, A. Joseph, S. Sastry, "A taxonomy of cyber attacks on SCADA systems," IEEE International Conference on Internet of Things, and Cyber, Physical and Social Computing, 2011.

[9] W. Mahoney, and R. A. Gandhi, "An integrated framework for control system simulation and regulatory compliance monitoring," Elsevier Intl. Journal of Critical Infrastructure Protection, vol. 4, no. 1, Apr 2011, pp. 41-53.

[10] B. Kesler, "The Vulnerability of Nuclear Facilities to Cyber Attack," Strategic Insights, vol. 10, no. 1, 2011.

[11] A. Almalawi, X. Yu, Z. Tari, and A. Fahad, "An unsupervised anomalybased detection approach for integrity attacks on SCADA systems," Elsevier Computers \& Security, vol. 46, Oct 2014, pp. 94-110.

[12] S. Backhaus, R. Bent, J. Bono, R. Lee, B. Tracey, D. Wolpert, D. Xie, and Y. Yildiz, "Cyber-physical security: A game theory model of humans interacting over control systems," arXiv:1304.3996 [cs.GT]

[13] D. S. Pidikiti, R. Kalluri, R. K. S. Kumar, and B. S. Bindhumadhava, "SCADA communication protocols: vulnerabilities, attacks and possible mitigations," CSIT, Mar 2013.

[14] Y. Yang, K. McLaughlin, T. Littler, S. Sezer, and H.F. Wang, "Rulebased intrusion detection system for SCADA networks," in Proc. $2^{\text {nd }}$ IET Conference in Renewable Power Generation, (RPG 2013), pp. 9-11, Sep 2013.

[15] P. Jain, and P Tripathi, "SCADA security: a review and enhancement for DNP3 based systems," CSIT, Dec 2013, 1(4): pp. 301-308. DOI 10.1007/s40012-013-0024-2. 
[16] R. Langner, "Stuxnet- Dissecting a cyberwarfare weapon," IEEE Security \& Privacy, vol. 9, no. 3, pp. 49-51, May 2011. DOI: 10.1109/MSP.2011.67.

[17] V. M. Igure, S. A. Laughter, and R. D. Williams, "Security issues in SCADA networks," Elsevier Computers \& Security, vol. 25, Oct 2006, pp. 498-506.

[18] White paper, "Web services security-The technology and its concerns, [online]. Available: https://www.acunetix.com/websitesecurity/webservices-wp/ http://www.acunetix.com/websitesecurity/web-services-wp/

[19] J. P. Disso, K. Jones, and S. Bailey, "A plausible solution to SCADA security honeypot systems," in Proc. 2013 Eighth Intl. Conf. on Broadband, Wireless Computing, Communication and Applications, Oct 2013.

[20] L. Constantin, "New Havex malware variants target industrial control system and SCADA users," PC World, Jun 2014.

[21] Symantec Security Response, "Dragonfly: Cyberespionage Attack Against Energy Suppliers", July 2014. [online]. Available: https://www.symantec.com/content/en/us/enterprise/media/security_resp onse/whitepapers/Dragonfly_Threat_Against_Western_Energy_Supplier s.pdf

[22] B. Genge, and C. Siaterlis, "Physical process resilience-aware network design for SCADA systems," Comput. Electr. Eng, vol. 40, no. 1, January 2014, pp. 142-157.

[23] Dell Security Annual Threat Report, [online]. Available: https://software.dell.com/docs/2015-dell-security-annual-threat-reportwhite-paper-15657.pdf

[24] I. N. Fovinoa, A. Carcanoa, M. Maseraa, and A. Trombettab, "An experimental investigation of malware attacks on SCADA systems," International Journal of Critical Infrastructure Protection, vol. 2, no. 4, Dec 2009, pp. 139-145.

[25] Information Security Management (ISMS), ISO/IEC 27001 [online]. Available: http://www.bsigroup.com/en-GB/iso-27001-information-security/

[26] K. Scarfone and M. Souppaya, NIST Special Publication 800-114, "User's Guide to Securing External Devices for Telework and Remote Access," Nov 2007.

[27] Guide to industrial control systems (ICS), NIST Special Publication 800-82, Revision 2, May 2015.

[28] A. Pauna, et al., "Window of exposure... a real problem for SCADA systems?" ENISA Recommendations for Europe on SCADA patching, Dec 2013.

[29] V. Urias, B. V. Leeuwen, and B. Richardson "Supervisory command and data acquisition (SCADA) system cyber security analysis using a Live,Virtual, and Constructive (LVC) testbed," MILCOM 2012 IEEE Military Communications Conference, Oct 2012, pp. 1-8.

[30] C. W. Ten, C.C. Liu, and G. Manimaran, "Vulnerability assessment of cybersecurity for SCADA systems," IEEE Transactions on Power Systems, vol. 23, no. 4, Nov 2008.

[31] K. Mathioudakis, N. Frangiadakis, A. Merentitis, and V. Gazis, "Towards generic SCADA simulators: A survey of existing multipurpose co-simulation platforms, best practices and use-cases,". [online]. Available: http://conf-scoop.org/ACE-2013/6_Kostas_ACE.pdf.

[32] K. Mets, J. A. Ojea, and C. Develder, "Combining power and communication network simulation for cost-effective smart grid analysis,", IEEE Communications Surveys \& Tutorials, vol. 16, no. 3, pp. 1771-1796, Mar 2014.

[33] Microsoft Security Bulletins, [online]. Available: https://technet.microsoft.com/en-us/security/bulletins.aspx

[34] T. August, R. August, and H. Shin, "Designing user incentives for cybersecurity," Communications of the ACM, vol. 57, no. 11, Nov 2014, pp. 43-46.

[35] P. Oman, and M. Phillips, "Intrusion detection and event monitoring in SCADA networks," Chapter Critical Infrastructure Protection, vol. 253 of the series IFIP International Federation for Information Processing, pp. 161-173.

[36] Aircrack-NG, [online]. Available: https://www.aircrack-ng.org/

[37] P. Huitsing, R. Chandia, M. Papa, and S. Shenoi, B, "Attack taxonomies for the Modbus protocols", Int. J. Critical Infrastructure Protection, vol. 1, pp. 37-44, Dec. 2008

[38] DNP-3 Protocol. [online]. Available: http://www.dnp.org/pages/aboutdefault.aspx.

[39] D. Jin, D. M. Nicol, and G. Yan, "An event buffer flooding attack in DNP3 controlled SCADA systems," in Proc. of Winter Simulation Conference, AZ, USA, 2011, pp. 2614-2626.
[40] A. Srivastava, T. Morris, T. Ernster, C. V. Shengyi, and U. Adhikari "Modeling cyber-physical vulnerability of the smart grid with incomplete information," IEEE Transactions on smart grid, vol. 4, no. 1, Mar 2013.

[41] J. Kirsch, S. Goose, Y. Amir, D. Wei, and P. Skare, "Survivable SCADA via intrusion-tolerant replication," IEEE Trans. on Smart Grid, vol. 5, no. 1, Jan 2014, pp. 60-70.

[42] J. Slay, and M. Miller, "Lessons learned from the Maroochy water breach," chapter Critical Infrastructure Protection, vol. 253 of the series IFIP International Federation for Information Processing, vol. 253, Springer Boston, pp. 73-82.

[43] B. Vijayan "Stuxnet renews power grid security concerns," Computerworld, Jul 26, 2010. [online]. Available: http://www.computerworld.com/article/2519574/security0/stuxnetrenews-power-grid-security-concerns.html

[44] P. Roberts, "SCADA vendors still need security wake-up call," [online]. Available: https://threatpost.com/scada-vendors-still-need-securitywake-call-102410/74603/

[45] IEEE Standard for Modeling and Simulation (M\&S) High Level Architecture (HLA) Framework and Rules, 1516.2-2010.

[46] R. Chabuksawar, B. Sinopoli, G. Karsai, A. Giani, A. Davies, H. Neems, and A. Davis "Simulation of network attacks on SCADA systems," First Workshop on Secure Control Systems, 2010.

[47] G. Hemingway, H. Neema, H. Nine, J. Sztipanovits, and G. Karsai, "Rapid synthesis of HLA-based heterogeneous simulation: A modelbased integration approach," Journal Simulation, vol. 88, no. 2, Feb 2012, pp. 217-232.

http://www2.engr.arizona.edu/ sprinkjm/research/c2wt/uploads/Internal Heterogeneous-Simulation.pdf

[48] J. J. Downs and E. F. Vogel. A plant-wide industrial process control problem. Computers \& Chemical Engineering, 17(3):245-255, 1993.

[49] C. Queiroz, A. Mahmood, and Z. Tari, "SCADASim-A framework for building SCADA simulations," IEEE Trans. on Smart Grid, vol. 2, issue 4, pp. 589-597, Sep 2011.

[50] D. Bytschkow, M. Zellner, and M. Duchon, "Combining SCADA, CIM, GridLab-D and AKKA for smart grid co-simulation," IEEE innovative Smart Grid Technologies Conference, Jun 2015.

[51] P. Novak, R. Šindelař, R. Mordinyi, "Integration framework for simulations and SCADA systems," Elsevier Simulation Modelling Practice and Theory, vol. 47, Sep 2014, pp. 121-140.

[52] C. W. Ten, G. Manimaran, C. C. Liu, "Cyber security for critical infrastructures: Attack and defence modelling," IEEE Trans. on Systems, Man, and Cybernetics-Part A: Systems and Humans, vol. 40, no. 4, Jul 2010

[53] Z. J. Zhang, Z. Yang, L. H. Zhu, L. Xiao, and L. Zhao, "A survey of SCADA test bed", Int. J. Wireless and Mobile Computing, vol. 8, no. 1, 2015, pp. 9-14

[54] A. Giani, G. Karsai, T. Roosta, A. Shah, B. Sinopoli, and J. Wiley, "A test bed for secure and robust SCADA systems," Special issue on the $14^{\text {th }}$ IEEE real-time and embedded technology and applications symposium, vol. 5, no. 2, Jul 2008.

[55] National SCADA Test Bed: Available online: http://energy.gov/sites/prod/files/oeprod/DocumentsandMedia/NSTB Fa ct Sheet FINAL 09-16-09.pdf

[56] A. Davies, G. Karsai, H. Neems, A. Giani, B. Sinopoli, and R. Chabuksawar "TRUST for SCADA: A simulation-based experimental platform," presentation [online]. Available: http://slideplayer.com/slide/3377726/

[57] A. A. Farooqui, S. S. H. Zaidi, A. Y. Memon, and S. Qazi, "Cyber security backdrop: A SCADA testbed," IEEE Conference on Computing, Communications and IT Applications, ComComAp 2014, Beijing, pp. 98-103, Oct 2014.

[58] T. Morris, R. Vaughn and Y. S. Dandass, "A testbed for SCADA control system cybersecurity research and pedagogy", in Proc. of the Seventh Annual Workshop on Cyber Security and Information Intelligence Research, 2011.

[59] A. Hahn, B. Kregel, M. Govindarasu, J. Fitzpatrick, R. Adnan, S. Sridhar and M. Higdon, "Development of the powercyber SCADA security testbed", in Proc. of the Sixth Annual Workshop on Cyber Security and Information Intelligence Research, 2010.

[60] G. Dondossola, F. Garrone and J. Szanto, "Supporting cyber risk assessment of power control system with experimental data," IEEE PCSE Power Systems Conference and Exposition Seattle, Washington USA, 15-18 March 2009. 
[61] A. Tesfahun, and D. L. Bhaskari, "A SCADA testbed for investigating cyber security vulnerabilities in critical infrastructures," Automatic Control and Computer Sciences, 2016, vol. 50, no. 1, pp. 54-62.

[62] C. N. Boldea, "SCADA virtual test environment development," [online] Available: journal.ro/includes/showArticle.php?identificatorArticol=310

[63] R. Leszczyna, I. N. Fovino, and M. Masera, "Simulating malware with MalSim" Journal in Computer Virology, vol. 6, no. 1, pp. 65-75, Feb 2008.

[64] E. Ciancamerla, M. Minichino, and S. Palmieri "Modeling cyber-attacks on a critical infrastructure scenario," Fourth International Conference on Information, Intelligence, Systems and Applications, IISA 2013, pp. 1-6, Jul 2013

[65] NETA: [online]. Available: www.omnetpp.org/component/content/article?id=3712:neta-release

[66] J. M. Moya, A. Araujo, Z. Bankovic, J. Goyeneche et al., "Improving security for SCADA sensor networks with reputation systems and SelfOrganizing Maps," Sensors 2009, vol. 9, pp. 9380-9397; doi:10.3390/s91109380.

[67] I. N. Fovino, A. Coletta, A. Carcano, and M. Masera, "Critical statebased filtering system for securing SCADA network protocols," IEEE Transactions on Industrial Electronics, vol. 59, no. 10, Oct 2012.

[68] F. Xie, Y. Peng, W. Zhao, X. Han, H. Li, R. Zhang, J. Zhao, and J. Liu, "Using simulation platform to analyze radio modem security in SCADA," 7th International Symposium on Resilient Control Systems (ISRCS), 2014, pp. 19-21 Aug. 2014.

[69] B. Schneier, “Attack Trees,” Dr. Dobb's Journal of Software Tools 24, Dec 1999, pp. 21-2.

[70] A. E. Bouchti, and A. Haqiq, "Modeling cyber-attack for SCADA systems using CoPNet Approach," International Conference on Complex Systems (ICCS), 2012, pp. 1-6, Nov 2012.

[71] A. P. Moore, R. J. Ellison, and R. C. Linger "Attack modelling for information security and survivability," Technical note, CMU/SEI-2001TN-001, 3-15-2001.Software Engineering Institute, Carnegie Mellon University.

[72] E.J. Byres, M. Franz, and D. Miller, "The use of attack trees in assessing vulnerabilities in SCADA systems," International Infrastructure Survivability Workshop (IISW'04), Lisbon, Portugal, 2004.

[73] C. W. Ten, C. C. Liu, and M. Govindarasu, "Vulnerability assessment of cybersecurity for SCADA systems using attack trees," IEEE Power Engineering Society General Meeting, Jun 2007.

[74] J. D. Markovic-Petrovic, and M. D. Stojanovic, "Analysis of SCADA system vulnerabilities to DDoS attacks," $11^{\text {th }}$ International Conference on Telecommunication in Modern Satellite, Cable and Broadcasting Service, TELSIKS, Oct 2013.

[75] A. A. C'ardenas, S. Amin, and S. Sastry, "Research challenges for the security of control systems," in Proc. of the 3rd conference on Hot topics in security, HOTSEC'08, Article No. 6.

[76] P. Maynard, K. McLaughlin, and B. Haberler "Towards understanding Man-in-the-middle attacks on IEC 60870-5-104 SCADA networks," International Symposium for ICS \& SCADA Cyber Security Research (ICS-CSR), St Polten, Austria, Sep 2014.

[77] G. Hug, and J. A. Giampapa, "Vulnerability assessment of AC state estimation with respect to false data injection cyber-attacks," IEEE Trans. On Smart Grid, vol. 3, no. 3, Sep 2012, pp. 1362-1370.

[78] N. M. Torrisi, O. Vukovic, G. Dan, and S. Hagdahl, "Peekaboo: A gray hole attack on encrypted SCADA communication using traffic analysis," IEEE International Conference on Smart Grid Communications, Nov 2014.

[79] W. Li, L. Xie, Z. Deng, and Z. Wang, "False sequential logic attack on SCADA system and its physical impact analysis," Computers \& Security, vol. 58, May 2016, pp. 149-159.

[80] Y. Mo, R. Chabukswar, B. Sinopoli, "Detecting integrity attacks on SCADA systems," IEEE Transactions on Control Systems Technology, vol. 22, no. 4, July 2014, pp. 1396-1407.

[81] S. Sridhar and G. Manimaran, "Data integrity attacks and their impacts on SCADA control system," IEEE PES General Meeting, pp. 1-6, Jul 2010

[82] F. Li, L. Shen, Y. Si, and J. Niu, "A method to enhance SCADA systems survivability through simulation technology," 2009 International Conference on Measuring Technology and Mechatronics Automation, ICMTMA, Apr 2009.

[83] M. Brand, C. Valli, and A. Woodward, "A Threat to Cyber Resilience: A malware rebirthing botnet," International Cyber Resilience conference, 2011.
[84] P. Baecher, M. Koetter, T. Holz, M. Dornseif, and F. Freiling, "The Nepenthes platform: An efficient approach to collect malware," LNCS 4219, pp. 165-184, 2006

[85] L. Yang, X. Cao, J. Li, A. Wang, W. Tan, Z. Yu, "Research on FNNbased security defence architecture model of SCADA network," in Proc. of IEEE $2^{\text {nd }}$ International Conference on Cloud Computing and Intelligent Systems, CCIS2012, Nov 2012.

[86] J. G. Süß, A. Pop, P. Fritzson, and L. Wildman, "Towards integrated model-driven testing of SCADA systems using the Eclipse modeling framework and Modelica," in Proc. of 19th Australian Conference on Software Engineering, pp. 149-159.

[87] C. Queiroz, A. Mahmood, and Z. Tari, "A probabilistic model to predict the survivability of SCADA systems," IEEE Transactions on Industrial Informatics, vol. 9, issue 4, pp. 1975-1985, Dec 2012.

[88] Y. Zhang, L. Wang, Y. Xiang, and C. W. Ten, "Power system reliability evaluation with SCADA cybersecurity considerations," IEEE Transactions on Smart Grid, vol. 6, no. 4, July 2015, pp. 1707-1721.

[89] Y. Cherdantseva, P. Burnap, A. Blyth, P. Eden, K. Jones, H. Soulsby, and K. Stoddart, "A review of cyber security risk assessment methods for SCADA systems", Computers and Security, vol. 56, Feb 2016, pp 127.

[90] M. H. Henry and Y. Y. Haimes, "A comprehensive network security risk model for process control networks," Risk Analysis, vol. 29, no. 2, 2009

[91] M.H. Henry, Minimax Envelopes for total cyber risk management in process control networks, Ph.D. Dissertation, Department of Systems and Information Engineering, University of Virginia, 2007.

[92] P.A.S. Ralston, J.H. Graham, and J.L. Hieb, "Cyber security risk assessment for SCADA and DCS networks," Elsevier ISA Transactions, vol. 46, issue 4, Oct 2007, pp. 583-594.

[93] A. Mahboob, and J. A. Zubairi, "Securing SCADA systems with open source software," 10th International Conference on High capacity Optical Networks and Enabling Technologies (HONET-CNS), Dec 2013.

[94] C. Phillips, and L. P. Swiler, "A graph-based system for networkvulnerability analysis," NSPW '98 in Proc. of the 1998 workshop on new security paradigms, pp. 71-79, 1998.

[95] nmap, [online]. Available: https://nmap.org/

[96] Metaspoilt, [online]. Available: https://www.metasploit.com/

[97] Sploitware [online]. Available: https://github.com/enaqx/sploitware

[98] J. Jiang, and L. Yasakethu, "Anomaly detection via one class SVM for protection of SCADA systems," International Conference on CyberEnabled Distributed Computing and Knowledge Discovery (CyberC), 2013.

[99] P. O'Kane, S. Sezer, K. McLaughlin, and E. G. Im, "SVM training phase reduction using dataset feature-filtering for malware detection," IEEE Transactions on Information Forensics and Security, vol. 8, no. 3, Mar 2013.

[100]P. Nader, P. Honeine, and P. Beauseroy, " $l_{p}$-norms in one-class classification for intrusion detection in SCADA systems," IEEE Transactions on Industrial Informatics, vol. 10, no. 4, Nov 2014, pp. 500-509

[101]Y. Simmhan, S. Aman, A. Kumbhare, R. Liu, S. Stevens, Q. Zhou, V. Prasanna, "Cloud-based software platform for big data analytics in smart grids," Computing in Science \& Engineering, vol. 15, issue 4, July-Aug. 2013, pp. 38-47.

[102]Ten Leading Practices for Smart Grid Analytics, Accenture Report. [online]. Available: https://www.accenture.com/gben/accenturesmartsolutions-sustainability.

[103]A. MacDermott, Q. Shi, M. Merabti and K. Kifayat, "Intrusion detection for critical infrastructure protection," 2012 PGNet.

[104] Quickdraw SCADA IDS. [Online]. Available: http://www.digitalbond.com, 2012.

[105]Y. Yang, K. McLaughlin, S. Sezer, Y.B. Yuan, and W. Huang, "Stateful intrusion detection for IEC 60870-5-104 SCADA security," IEEE PES General Meeting, Conference \& Exposition, 27-31 July 2014.

[106] K. Scarfone, and P. Mell, "Guide to Intrusion Detection and Prevention Systems (IDPS)," NIST Special Publication 800-94.

[107]M. Winn, M. Rice, S. Dunlap, J. Lopez and B. Mullins, "Constructing cost-effective and targetable industrial control system honeypots for production networks," Elsevier Intl. Journal of Critical Infrastructure Protection, vol. 10, issue C, Sep 2015, pp. 47-58.

[108] A. Carcano, A. Coletta, M. Guglielmi, M. Masera, I. Nai Fovino, and A. Trombetta, "A multidimensional critical state analysis for detecting intrusions in SCADA systems," IEEE Transactions on Industrial Informatics, vol. 7, no. 2, May 2011. 
[109] Snort, [online]. Available: https://www.snort.org/

[110] OSSEC, [online]. Available: http://ossec.github.io/

[111]Honeyd: Open source tool for creating Honeypots, [online]. Available: www.honeyd.org/

[112] V. H. Pham and M. Dacier, "Honeypot trace forensics: The observation viewpoint matters," Elsevier Future Generation Computer Systems, vol. 27, issue 5, May 2011, pp. 539-546.

[113] J. Goldenberg, Y. Shavitt, E. Shir, S. Solomon, "Distributive immunization of networks against viruses using the 'honey-pot' architecture," Nature physics, vol. 1, pp. 184-188, Dec 2005.

[114] OSSIM. [online]. Available: https://www.alienvault.com/products/ossim

[115]Good practices and recommendations for developing harmonised certification schemes, ENISA, Dec 2014.

[116]R.S.H. Piggin, "Securing SCADA in the cloud: Managing the risks to avoid the perfect storm," IET \& ISA $60^{\text {th }}$ International Instrumentation Symposium, Jun 2014.

[117] OpenSCADA, [online]. Available: http://openscada.org/

[118]R. Clarke, D. Dorwin, and R. Nash, "Is open source software more secure? [online]. Available: https://courses.cs.washington.edu/courses/csep590/05au/whitepaper_tur nin/oss(10).pdf

[119] W. Wu, J. Gao, Y. Yuan, H. Zhao, and K. Chang "Water distribution network real-time simulation based on SCADA system using OPC communication," Networking, Sensing and Control (ICNSC), 2011 IEEE International Conference on, Delft, 2011, pp. 329-334. doi: 10.1109/ICNSC.2011.5874916.

[120]S. H. Leitner, and W. Mahnke, "OPC UA - Service-oriented architecture for industrial applications," [online]. Available: http://pi.informatik.unisiegen.de/stt/26_4/01_Fachgruppenberichte/ORA2006/07_leitnerfinal.pdf

[121]S. Cavalieri, G. Cutuli and S. Monteleone, "Evaluating impact of security on OPC UA performance," 3rd Conference on Human System Interactions (HSI), 2010, 23 July 2010.

[122]K. Stouffer, NIST Briefing: ICS Cybersecurity Guidance - NIST SP 800-82, Guide to ICS Security, Aug 2013. 


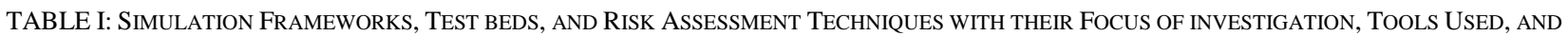
APPLICATION.

\begin{tabular}{|c|c|c|c|c|c|}
\hline Category & Sub-category & Focus & Tools used & Application & Citations \\
\hline \multirow{6}{*}{$\begin{array}{l}\text { Simulation } \\
\text { framework }\end{array}$} & SCADASiM & Regulatory monitoring & ADACS & Water supply system & [9] \\
\hline & SCADAsim & DDoS, spoofing attacks & OMNeT++ & $\begin{array}{l}\text { Smart meters, wind power } \\
\text { plant }\end{array}$ & [49] \\
\hline & MAlsim & $\begin{array}{l}\text { Malware simulation for } \\
\text { security evaluation }\end{array}$ & MAlsim based on JADE & Power plant & [63] \\
\hline & Co-simulation & $\begin{array}{l}\text { Custom smart } \\
\text { component analysis }\end{array}$ & $\begin{array}{l}\text { CIM, GridLab-D, AKKA, } \\
\text { EclipseSCADA }\end{array}$ & Power system & [50] \\
\hline & Framework & $\begin{array}{l}\text { Integration of simulations } \\
\text { and SCADA }\end{array}$ & EngSB & Software prototype & {$[51]$} \\
\hline & Framework & Malware experimentation & MATLAB, Emulab & $\begin{array}{l}\text { Stuxnet on a power plant, } \\
\text { Tennessee-Eastman }\end{array}$ & [6] \\
\hline \multirow{10}{*}{ Test bed } & Simulation & DoS & $\begin{array}{l}\text { TrueTime } \\
\text { (MATLAB/Simulink) }\end{array}$ & $\begin{array}{l}\text { American Gas Association } \\
\text { Report No. } 12\end{array}$ & [57] \\
\hline & $\begin{array}{l}\text { Hybrid } \\
\text { (simulation, } \\
\text { emulation, } \\
\text { hardware) }\end{array}$ & $\begin{array}{l}\text { Anomaly based detection } \\
\text { for HMI attack, DoS }\end{array}$ & $\begin{array}{l}\text { OPNET, } \quad \text { PowerWorld, } \\
\text { ASPS }\end{array}$ & $\begin{array}{l}\text { Biosphere } 2 \text { Power } \\
\text { Grid at the University of } \\
\text { Arizona }\end{array}$ & [5] \\
\hline & Pedagogy & Modbus, HMI & Hardware, software, Snort & $\begin{array}{l}\text { Various industrial } \\
\text { applications }\end{array}$ & {$[58]$} \\
\hline & $\begin{array}{l}\text { Intrusion and } \\
\text { Defence }\end{array}$ & $\begin{array}{l}\text { Communication protocols, } \\
\text { networks }\end{array}$ & $\begin{array}{l}\text { Software, hardware, } \\
\text { anomaly detection }\end{array}$ & PowerCyber testbed & [59] \\
\hline & $\begin{array}{l}\text { Statistical data } \\
\text { gathering }\end{array}$ & DoS, Data logging & Data statistics & $\begin{array}{l}\text { Power Control } \\
\text { Systems - Resilience } \\
\text { Testing Laboratory of } \\
\text { CESI-RICERCA }\end{array}$ & {$[60]$} \\
\hline & Attack & DoS, integrity, phishing & Simulation & $\begin{array}{l}\text { Single simulation-based } \\
\text { instantiation }\end{array}$ & [54] \\
\hline & $\begin{array}{l}\text { Communications } \\
\text { network }\end{array}$ & DDoS, Network & $\begin{array}{l}\text { Simulink/Stateflow, HLA, } \\
\text { NS2, OPNET, OMNeT++ }\end{array}$ & $\begin{array}{l}\text { Tennessee } \\
\text { chemical process }\end{array}$ & [56] \\
\hline & Attack & DDoS & $\begin{array}{lcc}\text { SCADA } & 2, & \text { Modbus } \\
\text { simulator, GNS3 } & \\
\end{array}$ & Generic implementation & {$[62]$} \\
\hline & Virtual machine & $\begin{array}{l}\text { Modbus TCP/IP, DDoS, } \\
\text { False data injection }\end{array}$ & $\begin{array}{l}\text { Common Open } \begin{array}{r}\text { Research } \\
\text { Emulator, }\end{array} \\
\text { Pymodbus, Ettercap }\end{array}$ & Water tank system & {$[61]$} \\
\hline & $\begin{array}{l}\text { Intrusion } \\
\text { detection, event } \\
\text { monitoring }\end{array}$ & RTUs & Snort, Perl, XML & Electric substation & [35] \\
\hline \multirow{5}{*}{$\begin{array}{c}\text { Risk } \\
\text { assessment }\end{array}$} & Probabilistic & Risk estimation & HMM, IIM, RFRM & Generic system & {$[92]$} \\
\hline & Modeling & Control systems & NSRM & Oil pipeline pump station & {$[90]$} \\
\hline & $\begin{array}{l}\text { Network } \\
\text { vulnerability }\end{array}$ & Graph & Attack graph & Simplified example & {$[94]$} \\
\hline & Attack graphs & Bayesian network & MTTC, CVSS & IEEE RTS79 & {$[88]$} \\
\hline & Attack trees & Vulnerability assessment & Vulnerability index & Control centre & {$[73]$} \\
\hline
\end{tabular}




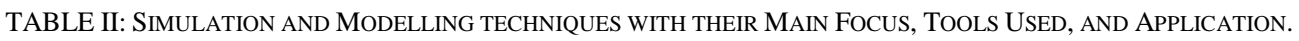

\begin{tabular}{|c|c|c|c|c|c|}
\hline Category & Sub-category & Focus & Tools used & Application & Citations \\
\hline \multirow{9}{*}{ Simulation } & Attack trees & Intrusion & Colored Petri Net & SCADA case study & [70] \\
\hline & $\begin{array}{l}\text { False sequential } \\
\text { logic attack }\end{array}$ & Intrusion & MATLAB/Simulink & Three tank system & [79] \\
\hline & Malware attacks & Modbus & MAlsim & Power plant testbed & [24] \\
\hline & Test bed & Modbus TCP & $\begin{array}{l}\text { OSSIM, Snort, Sebekd, } \\
\text { OpenVAS }\end{array}$ & Simulated PLC & [93] \\
\hline & MITM & IEC 60870-5-104 & $\begin{array}{l}\text { Snort, Qtester, Kali Linux, } \\
\text { WinPP104 }\end{array}$ & $\begin{array}{l}\text { Software simulated } \\
\text { laboratory, testbed }\end{array}$ & [76] \\
\hline & Graph & Modbus & ' $\mathrm{C}$ ' Language & $\begin{array}{l}\text { Tennessee Eastman } \\
\text { chemical process }\end{array}$ & [22] \\
\hline & Monitoring & Malicious commands & $\begin{array}{l}\text { Real-time and simulation } \\
\text { monitor }\end{array}$ & Water treatment plant & [82] \\
\hline & Attack & DDoS & OPNET & Hydropower & [74] \\
\hline & Attack & $\begin{array}{l}\text { Malware injection, } \\
\text { DoS, MITM }\end{array}$ & Netlogo & $\begin{array}{l}\text { Electrical grid connected } \\
\text { to corporate network, } \\
\text { NS2 }\end{array}$ & [64] \\
\hline \multirow{6}{*}{ Modelling } & Mathematical & DoS, deception attacks & Linear dynamical models & Water tank & [75] \\
\hline & Mathematical & Model-driven testing & Modelica, Eclipse & $\begin{array}{l}\text { Tank with valves and } \\
\text { pumps }\end{array}$ & [86] \\
\hline & Game Theory & $\begin{array}{l}\text { Interactions between } \\
\text { cyber intruder and } \\
\text { operator }\end{array}$ & $\begin{array}{l}\text { Semi network-form game } \\
\text { (SNFG) }\end{array}$ & Distribution feeder line & [12] \\
\hline & Bayesian Networks & System survivability & SCADASim & MITM & [87] \\
\hline & FNN & Defence & Factor neural network & Generic & {$[85]$} \\
\hline & $\begin{array}{l}\text { Framework, attack } \\
\text { trees }\end{array}$ & Modbus, DoS & RAIM & $\begin{array}{l}\text { power system control } \\
\text { network }\end{array}$ & [52] \\
\hline \multirow{3}{*}{ Attack } & Attack trees & Modbus/tcp & Attack trees & Generic system & [72] \\
\hline & Replay attack & sensors & Analytical, simulation & $\begin{array}{l}\text { Tenessee } \\
\text { problem }\end{array}$ & {$[80]$} \\
\hline & False data injection & Ac state estimation & Analytics & IEEE 57 bus system & [77] \\
\hline
\end{tabular}


TABLE III: TOOLS AND TECHNIQUES WITH THEIR MAIN FoCUS, TOOLS USED AND APPLICATION.

\begin{tabular}{|c|c|c|c|c|c|}
\hline Category & subcat & Focus & Tools Used & Application & Citations \\
\hline \multirow{7}{*}{$\begin{array}{l}\text { Machine } \\
\text { Learning }\end{array}$} & Anomaly based & $\begin{array}{l}\text { Integrity attacks, } \\
\text { Modbus/TCP }\end{array}$ & $\begin{array}{l}\text { WEKA, EPANET, } \\
\text { VMs, } k \text {-means }\end{array}$ & $\begin{array}{l}\text { Simulated and } \\
\text { real data sets, } \\
\text { Water distribution } \\
\text { system }\end{array}$ & [11] \\
\hline & Anomaly detection & Telecommunications networks & SVM & Data sets & [98] \\
\hline & Feature filtering & Malware detection & 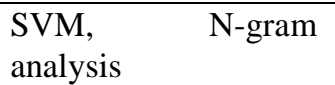 & Generic & [99] \\
\hline & $\begin{array}{ll}\text { One } & \text { class } \\
\text { classification } & \end{array}$ & Malware intrusions & SVDD, kernel PCA & $\begin{array}{l}\text { Real data from } \\
\text { gas pipeline } \\
\text { testbed and water } \\
\text { treatment plant }\end{array}$ & [100] \\
\hline & $\begin{array}{l}\text { Reputation system, } \\
\text { distributed agents }\end{array}$ & Sensors & $\begin{array}{l}\text { Unsupervised } \\
\text { learning, SOM }\end{array}$ & Sensor networks & [66] \\
\hline & $\begin{array}{l}\text { Communication } \\
\text { protocols }\end{array}$ & DNP3, gray hole attack & SVM & $\begin{array}{ll}\text { Trace } & \text { based } \\
\text { simulation } & \end{array}$ & [78] \\
\hline & Analytics & Cloud based data analytics & $\begin{array}{l}\text { OpenPlanet, Hadoop, } \\
\text { regression tree, Floe, } \\
\text { MapReduce, WEKA, } \\
\text { VM }\end{array}$ & $\begin{array}{lr}\text { Los } & \text { Angeles } \\
\text { Smart } & \text { Grid } \\
\text { Project } & \end{array}$ & [101] \\
\hline \multirow{4}{*}{$\begin{array}{l}\text { Intrusion } \\
\text { Detection } \\
\text { System }\end{array}$} & Rule based & IEC 60870-5-104 & $\begin{array}{lr}\text { Deep r packet } \\
\text { inspection, ITACA }\end{array}$ & $\begin{array}{ll}\text { Protocol traffic } \\
\text { case study }\end{array}$ & [14] \\
\hline & Filtering system & Modbus and DNP311 & Firewall & $\begin{array}{l}\text { Industrial } \\
\text { Network Security } \\
\text { Laboratory }\end{array}$ & [67] \\
\hline & $\begin{array}{lr}\text { Intrusion } & \text { tolerance, } \\
\text { state } & \text { machine } \\
\text { approach } & \end{array}$ & SCADA master & $\begin{array}{l}\text { Hardware, } \\
\text { replication }\end{array}$ & $\begin{array}{l}\text { Simple SCADA } \\
\text { master, and RDU } \\
\text { for electricity } \\
\text { transmission and } \\
\text { distribution }\end{array}$ & [41] \\
\hline & Critical state based & Modbus on PLC & ISML & $\begin{array}{l}\text { Joint Research } \\
\text { Centre testbed, }\end{array}$ & [108] \\
\hline \multirow{5}{*}{ Honey Pots } & Botnets & Detect new botnets & honeyd & Data traces & {$[112]$} \\
\hline & Analysis & $\begin{array}{l}\text { Protection to SCADA, anti- } \\
\text { honeypot techniques }\end{array}$ & Honeywell CDROM & $\begin{array}{l}\text { Anti-honeypot } \\
\text { techniques }\end{array}$ & [19] \\
\hline & Using proxy & $\begin{array}{l}\text { Techniques for low cost honey } \\
\text { pots }\end{array}$ & $\begin{array}{l}\text { Honeyd+, Raspberry } \\
\text { Pi }\end{array}$ & $\begin{array}{l}\text { Study Slammer, } \\
\text { Code Red, Blaster }\end{array}$ & [107] \\
\hline & Malware & Large scale malware collection & nepenthes & nepenthes & [84] \\
\hline & Virus & $\begin{array}{l}\text { Virus discovery and fast antivirus } \\
\text { dissemination }\end{array}$ & $\begin{array}{l}\text { Dynamic distributed } \\
\text { immunisation } \\
\text { strategy }\end{array}$ & Email network & [113] \\
\hline Post attack & Forensics & Smart grids & $\begin{array}{l}\text { Traces, } \\
\text { system }\end{array}$ & Attack attribution & [7], [112] \\
\hline
\end{tabular}

